\title{
Stochastic Formulation for a Partial Neural Circuit of C. elegans
}

\author{
YUISHI IWASAKI \\ Department of Systems Engineering, \\ Ibaraki University, \\ Hitachi, Ibaraki 316-8511, \\ Japan \\ E-mail: iwasaki@mx.ibaraki.ac.jp \\ SOHEI GOMI \\ Faculty of Humanities and Social Sciences, \\ Iwate University, \\ Morioka, Iwate 020-8550, \\ Japan \\ E-mail: gomi@iwate-u.ac.jp
}

\begin{abstract}
We present a stochastic formulation for a partial neural circuit of Caenorhabditis elegans. This study is concerned with how to reduce the degree of freedom in a large neural circuit. In the presented formulation, neurons in the whole neural circuit are divided into two complementary groups. One is the neurons which are mainly associated with a certain behavior, and the other is the remaining neurons of $C$. elegans. In an ordinary study on a partial neural circuit, the influence of the latter (the remaining neurons) on the former (the associated neurons) is completely neglected. In the presented formulation, however, the influence is expressed by a stochastic variable. The structure of the ensemble for the stochastic variable is appropriately evaluated by the neural connectivity of $C$. elegans since the neural connectivity of $C$. elegans has been completely determined. In this way, the degree of freedom is effectively reduced. We apply the presented formulation to determine the synaptic signs in the touch sensitivity circuit of $C$. elegans. The synaptic signs are determined to satisfy the locomotory behaviors in $C$. elegans. We find that the influence of the remaining neurons on the touch sensitivity circuit is important to determine the synaptic signs.
\end{abstract}

Note: Bulletin of Mathematical Biology, Vol.66, Issue.4 (2004) 727-743. 


\section{INTRODUCTION}

From the viewpoint of mathematical neurobiology, the nematode Caenorhabditis elegans is a useful model organism because of the following reasons. Firstly, the neural connectivity of $C$. elegans has been completely determined (Albertson and Thomson, 1976; White et al., 1986). Some databases of the neural connectivity are available for computational use (Achacoso and Yamamoto, 1992; Oshio et al., 1998; Durbin, not published as a real paper). Secondly, there are a number of experimental studies on several behaviors and learning not only for the wild-type but also for the mutants of C. elegans (Wood 1988; Riddle et al., 1997). Thirdly, the neurons which are mainly associated with the behaviors have been identified.

The hermaphrodite C. elegans has 302 neurons. There are about 5800 chemical synapses and about 1400 gap junctions between the neurons (Oshio et al., 1998). To understand neural information processing in $C$. elegans, we usually focus on a partial neural circuit instead of the whole neural circuit. On the basis of the above mentioned knowledge on C. elegans, computational studies on the partial neural circuits are carried out (Wicks et al., 1996; Cangelosi and Parisi, 1997; Ferree and Lockery, 1999; Majewska and Yuste, 2001). In these studies, the partial neural circuits are assumed to be closed. That is, only the associated neurons and their connectivity are taken into account. The influence of the remaining neurons of $C$. elegans is completely neglected to reduce the degree of freedom in neural modeling. However, there is no guarantee that a result in the closed partial neural circuit is consistent with that in the whole (real) neural circuit since neural information processing is a highly nonlinear phenomenon. Although laser ablation experiments of the neuron have been performed on C. elegans to identify the associated neurons, this is still a problem in neural modeling.

Our main purpose is to propose a mathematical framework for studying a large biological neural circuit, which enables us to consider the influence of the neurons except those in the partial neural circuit. We apply this framework to solve a problem of studying the 'synaptic sign' (neurotransmitter phenotype) in the neural circuit of $C$. elegans. There are two types of connections between neurons; chemical synapse and gap junction. A chemical synapse is characterized by the synaptic sign; whether a chemical synapse is functionally excitatory or inhibitory. The synaptic signs of $C$. elegans are not fully identified since the electrophysiological measurement is difficult. In a computational study on the synaptic sign, a mathematical model of the nervous system is introduced at first. Secondly, the synaptic signs in the model are determined to satisfy some behavioral criteria. According to these procedures, the synaptic signs in the tap withdrawal circuit (Wicks et al., 1996) and the synaptic signs in the touch sensitivity circuit (Majewska and Yuste, 2001) have been studied. The synaptic signs in Majewska and Yuste (2001) do not satisfy the Dale's principle (Dale, 1935). The Dale's principle is a hypothesis that a neuron releases the same neurotransmitter at all its synapses. Furthermore, a neurotransmitter has been supposed to cause either postsynaptic excitation or inhibition, but not both. Although there is evidence that a neuron can release more than one neurotransmitter, and that a single neurotransmitter can have both functionally excitatory and inhibitory effects (McIntire et al., 1993; Dent et al., 1997; Kandel et al., 2000; Alberts et al., 2002), these occurrences might be rare. In the presented study, we assume that all synaptic connections from the same neuron have the same synaptic sign. 
Our conditions to study the synaptic signs of C. elegans are the following. (i) There are a large number of chemical synapses (the degree of freedom) between the neurons in the whole neural circuit. (ii) Therefore only a small number of the neurons are considered in practice. (iii) The influence of the remaining neurons in the whole neural circuit could not be fully taken into account. The Langevin equation (Langevin, 1908; Uhlenbeck and Ornstein, 1930), which is popular in a wide area of physical systems, give us a prescription under such conditions. The Langevin equation is the equation of motion for the Brownian particle of the mass $m$ moving with the velocity $v$ in a surrounding medium; $m(d v / d t)=-\gamma v+R$. Here $\gamma$ is the friction constant and $R$ is the random 'fluctuating force'. In the right-hand side of the Langevin equation, the forces exerted by the medium are conceptually divided into two parts. One is the friction term $-\gamma v$ which represents the 'systematic' part of the interactions between the Brownian particle and the medium. The other is the random force $R$ which represents the remaining 'nonsystematic' part of the molecular interactions. For the nonsystematic part, the stochastic property is implicitly expressed in $R$ instead of the details. Since the medium contains a huge number of molecules, all the molecular interactions are not taken into account explicitly in the Langevin equation.

Motivated by the above mentioned situations, we present a stochastic formulation for a partial neural circuit of $C$. elegans in Section 2. In the presented formulation, the neurons in the whole neural circuit are divided into two groups such as in the Langevin equation. One is the neurons which are mainly associated with a certain behaviors of $C$. elegans, and the other is the remaining neurons. The former is explicitly considered in neural modeling. To reduce the degree of freedom in neural modeling, on the other hand, the influence of the latter on the former is expressed by a stochastic variable. In the case of $C$. elegans, the structure of the ensemble for the stochastic variable is appropriately evaluated by the neural connectivity. In Section 3, we apply the presented formulation to determine the synaptic signs in the touch sensitivity circuit. We also examine the effect of the stochastic variable on the numerical results. In Section 4, we draw the conclusions.

\section{Stochastic Framework in Neural Modeling}

\subsection{Reduction of the degree of freedom.}

Generally, state of neuron is governed by the total inputs from other neurons. This is expressed by

$$
\text { state of } i \text { th neuron }=F_{i}\left(\sum_{j} n_{i j}^{\text {chem }} I_{i j}^{\text {chem }}+\sum_{j} n_{i j}^{\text {gap }} I_{i j}^{\text {gap }}+I_{i}^{\text {rest }}\right) .
$$

Here $I_{i j}^{\text {chem }}$ and $I_{i j}^{\text {gap }}$ represent inputs from the $j$ th neuron to the $i$ th neuron through a chemical synapse and a gap junction, respectively. The $I_{i}^{\text {rest }}$ represents other input caused by sensory stimulation or external current injection. The $n_{i j}^{\text {chem }}$ and $n_{i j}^{\text {gap }}$ represent the total number of chemical synapses and gap junctions from the $j$ th neuron to the $i$ th neuron, respectively. Since a gap junction is a bidirectional connection, $n_{i j}^{\text {gap }}=n_{j i}^{\text {gap }}$ for any pair of $i$ and $j$. On the other hand, $n_{i j}^{\text {chem }} \neq n_{j i}^{\text {chem }}$ since a chemical synapse is a unidirectional connection. The $F_{i}$ is a certain 
function which depends on a choice of neural model. In the cable equation (Koch and Segev, 1989), for example, the left-hand side of equation ( 1 ) is the rate of change of a neuron's membrane potential, and the function is simply $F_{i}(x)=x$. In equation $(1)$, we assume that the electrical signals are proportional to the number of the connections $n_{i j}^{\text {chem }}$ and $n_{i j}^{\text {gap }}$.

In equation (1), the sum in respect of $j$ is taken for all connected neurons. As the first step in the presented framework, neurons in a whole nervous system are divided into two complementary groups. One is the neurons $\mathcal{G}$ in a given partial circuit which is the subject of study. The other is the remaining neurons $\mathcal{G}^{\mathrm{c}}$ which is the complement of $\mathcal{G}$. In set theory, $\mathcal{G}^{\mathrm{c}}=1-\mathcal{G}$. In a study on behavior of $C$. elegans, for example, $\mathcal{G}$ are the neurons which are mainly associated with a certain behavior. As the second step, the total inputs in equation (1) are divided into those from the neurons $\mathcal{G}$ and those from the neurons $\mathcal{G}^{\mathrm{c}}$. When the sum of inputs from the neurons $\mathcal{G}^{\mathrm{c}}$ is denoted by $R_{i}$, equation (1) becomes

$$
\begin{aligned}
\text { state of } i \text {-th neuron } & =F_{i}\left(\sum_{j \in \mathcal{G}} n_{i j}^{\text {chem }} I_{i j}^{\text {chem }}+\sum_{j \in \mathcal{G}} n_{i j}^{\text {gap }} I_{i j}^{\text {gap }}+I_{i}^{\text {rest }}+R_{i}\right), \\
R_{i} & =\sum_{j \in \mathcal{G}^{\mathrm{c}}} n_{i j}^{\text {chem }} I_{i j}^{\text {chem }}+\sum_{j \in \mathcal{G}^{\mathrm{c}}} n_{i j}^{\text {gap }} I_{i j}^{\text {gap }} .
\end{aligned}
$$

As the third step, a stochastic framework is applied to $R_{i}$ to reduce the degree of freedom in equation (2). Instead of the details of the neurons $\mathcal{G}^{\mathrm{c}}$, a statistical property of the neurons $\mathcal{G}^{\mathrm{c}}$ is extracted in $R_{i}$ under a certain assumption. In the presented study, $I_{i j}^{\text {chem }}$ and $I_{i j}^{\text {gap }}$ in equation (3) are assumed to be mutually independent stochastic variables; however this assumption is not rigorously valid in the nervous system. Once this assumption is accepted as a working hypothesis, the central limit theorem derives that $R_{i}$ is a stochastic variable chosen from the Gaussian distribution as $j \rightarrow \infty$.

$$
R_{i} \sim \mathrm{N}\left(\mu_{i}, \sigma_{i}^{2}\right) .
$$

Equations (2) and (4) indicate that $R_{i}$ plays the role of an 'external noise' to the neurons $\mathcal{G}$. In the case of $C$. elegans, $n_{i j}^{\text {chem }}$ and $n_{i j}^{\text {gap }}$ have been determined for any pair of neurons. The stochastic properties of $R_{i}$, that is, the average $\mu_{i}$ and the variance $\sigma_{i}^{2}$, can be estimated from $n_{i j}^{\text {chem }}$ and $n_{i j}^{\text {gap }}\left(i \in \mathcal{G}, j \in \mathcal{G}^{\text {c }}\right)$.

State of the neurons $\mathcal{G}$ is explicitly given by equation (2). In this sense, the first and second terms of the right-hand side of equation (2) are the 'systematic' part of the inputs. On the other hand, $R_{i}$ is the 'nonsystematic' part of the inputs in the sense that the details of the neurons $\mathcal{G}^{\mathrm{c}}$ are not considered in the neural modeling.

\subsection{Application to McCulloch-Pitts model.}

The presented framework is applied to the McCulloch-Pitts model (McCulloch and Pitts, 1943). The McCulloch-Pitts model takes an extremely simplified representation of real neural properties, and has been extended in various ways. Since the presented study is motivated not physiologically but mathematically as mentioned in Section 1, the McCulloch-Pitts model is chosen for the sake of mathematical simplicity. In the McCulloch-Pitts model, a signal unidirectionally propagates to a connected neuron if and only if the neuron is active. This picture 
is suitable for the chemical synapse. On the other hand, the gap junction is considered to be a passive bidirectional connection between neurons. Since about $20 \%$ of the neural connections of $C$. elegans are gap junctions, the McCulloch-Pitts model should be modified to represent the nervous system of $C$. elegans. In the existence of both the chemical synapses and the gap junctions, activation of the neuron is governed by the following equation in the presented study.

$$
x_{i}(n+1)=\theta\left(\sum_{j} w_{i j}^{\text {chem }} n_{i j}^{\text {chem }} x_{j}(n)+g \sum_{j} n_{i j}^{\text {gap }}\left(x_{j}(n)-x_{i}(n)\right)-C_{i}\right) .
$$

Here $x_{i}(n)$ is the binary state of the $i$-th neuron at time step $n ; x_{i}(n)=1$ for active state and $x_{i}(n)=0$ for inactive state. The $w_{i j}^{\text {chem }}$ is the synaptic sign; $w_{i j}^{\text {chem }}=+1$ for an excitatory chemical synapse and $w_{i j}^{\text {chem }}=-1$ for an inhibitory chemical synapse. The $\theta(x)$ is the step function; $\theta(x)=1$ if $x>0$ and $\theta(x)=0$ if $x \leq 0$. The $g$ is the ratio between 'weight' of a chemical synapse and that of a gap junction. The $C_{i}$ is the voltage threshold. The first term in the right-hand side of equation (5) represents the sum of inputs through the chemical synapse which is formulated according to the original McCulloch-Pitts model. The second term in the right-hand side of equation (5) represents the sum of inputs through the gap junction. In the cable equation, a gap junction is formulated as an ohmic resistance. That is, current flows in proportion to the difference of the membrane potentials between the neurons. Considering the correspondence between the membrane potential in the cable equation and $x_{i}(n)$ in the McCulloch-Pitts model, the difference between the membrane potentials is simply replaced with $\left(x_{j}(n)-x_{i}(n)\right)$ in equation (5).

According to the second step in the presented formulation, the total inputs in equation (5) are divided into those from the neurons $\mathcal{G}$ and those from the neurons $\mathcal{G}^{\mathrm{c}}$.

$$
\begin{gathered}
x_{i}(n+1)=\theta\left(\sum_{j \in \mathcal{G}} w_{i j}^{\text {chem }} n_{i j}^{\text {chem }} x_{j}(n)+g \sum_{j \in \mathcal{G}} n_{i j}^{\text {gap }}\left(x_{j}(n)-x_{i}(n)\right)-C_{i}+R_{i}^{\text {chem }}+g R_{i}^{\text {gap }}\right) . \\
R_{i}^{\text {chem }}=\sum_{j \in \mathcal{G}^{\mathrm{c}}} w_{i j}^{\text {chem }} n_{i j}^{\text {chem }} x_{j}(n), \\
R_{i}^{\text {gap }}=\sum_{j \in \mathcal{G}^{\mathrm{c}}} n_{i j}^{\text {gap }}\left(x_{j}(n)-x_{i}(n)\right) .
\end{gathered}
$$

The $R_{i}^{\text {chem }}$ and $R_{i}^{\text {gap }}$ represent the sum of the inputs from the neurons $\mathcal{G}^{\text {c }}$.

According to the third step in the presented formulation, $w_{i j}^{\text {chem }}$ and $x_{j}(n)$ in equations $(7)$ and (8) are assumed to be mutually independent stochastic variables. That is, for any $j \in \mathcal{G}^{\mathrm{c}}$,

$$
\begin{gathered}
w_{i j}^{\text {chem }}= \begin{cases}+1 & \text { with probability } p_{i j}, \\
-1 & \text { with probability } 1-p_{i j},\end{cases} \\
x_{j}(n)= \begin{cases}1 & \text { with probability } q_{j}, \\
0 & \text { with probability } 1-q_{j} .\end{cases}
\end{gathered}
$$

When the central limit theorem is naively applied to equation $(7), R_{i}^{\text {chem }}$ becomes a stochastic variable chosen from the Gaussian distribution whose average is $\mu_{i}^{\text {chem }}$ and variance is $\left(\sigma_{i}^{\text {chem }}\right)^{2}$. 


$$
\begin{gathered}
R_{i}^{\text {chem }} \sim \mathrm{N}\left(\mu_{i}^{\text {chem }},\left(\sigma_{i}^{\text {chem }}\right)^{2}\right), \\
\mu_{i}^{\text {chem }}=\sum_{j \in \mathcal{G}^{\mathrm{c}}} q_{j}\left(2 p_{i j}-1\right) n_{i j}^{\text {chem }}, \\
\left(\sigma_{i}^{\text {chem }}\right)^{2}=\sum_{j \in \mathcal{G}^{\mathrm{c}}} q_{j}\left(1-q_{j}\left(2 p_{i j}-1\right)^{2}\right)\left(n_{i j}^{\text {chem }}\right)^{2} .
\end{gathered}
$$

In addition to equation $(10), x_{i}(n)$ in equation (8) is assumed to be a stochastic variable which is mutually independent of $x_{j}(n)$. That is, for any $x_{i}(n)$ in equation (8),

$$
x_{i}(n)= \begin{cases}1 & \text { with probability } r_{i}, \\ 0 & \text { with probability } 1-r_{i} .\end{cases}
$$

When the central limit theorem is naively applied to equation (8), $R_{i}^{\text {gap }}$ becomes a stochastic variable chosen from the Gaussian distribution whose average is $\mu_{i}^{\text {gap }}$ and variance is $\left(\sigma_{i}^{\text {gap }}\right)^{2}$.

$$
\begin{gathered}
R_{i}^{\text {gap }} \sim \mathrm{N}\left(\mu_{i}^{\text {gap }},\left(\sigma_{i}^{\text {gap }}\right)^{2}\right), \\
\mu_{i}^{\text {gap }}=\sum_{j \in \mathcal{G}^{\text {c }}}\left(q_{j}-r_{i}\right) n_{i j}^{\text {gap }}, \\
\left(\sigma_{i}^{\text {gap }}\right)^{2}=\sum_{j \in \mathcal{G}^{\text {c }}}\left(q_{j}\left(1-q_{j}\right)+r_{i}\left(1-r_{i}\right)\right)\left(n_{i j}^{\text {gap }}\right)^{2} .
\end{gathered}
$$

Here an appropriate description of $R_{i}^{\text {gap }}$ for $C$. elegans must be noted. Any $x_{i}(n)(i \in \mathcal{G})$ is explicitly given by equation (6) at any time step $n$ and $n_{i j}^{\text {gap }}$ of $C$. elegans is determined for any pair of $i$ and $j$. Only $x_{j}(n)\left(j \in \mathcal{G}^{\mathrm{c}}\right)$ is not given in the right-hand side of equation (8). It is appropriate that $R_{i}^{\text {gap }}=\tilde{R}_{i}^{\text {gap }}-x_{i}(n) \sum_{j \in \mathcal{G}^{\mathrm{c}}} n_{i j}^{\text {gap }}$ and only $\tilde{R}_{i}^{\text {gap }}=\sum_{j \in \mathcal{G}^{\mathrm{c}}} n_{i j}^{\text {gap }} x_{j}(n)$ should be a stochastic variable. From equation $(10)$, we obtain that $\tilde{R}_{i}^{\text {gap }} \sim \mathrm{N}\left(\tilde{\mu}_{i}^{\text {gap }},\left(\tilde{\sigma}_{i}^{\text {gap }}\right)^{2}\right)$, $\tilde{\mu}_{i}^{\text {gap }}=\sum_{j \in \mathcal{G}^{\mathrm{c}}} q_{j} n_{i j}^{\text {gap }}$ and $\left(\tilde{\sigma}_{i}^{\text {gap }}\right)^{2}=\sum_{j \in \mathcal{G}^{\mathrm{c}}} q_{j}\left(1-q_{j}\right)\left(n_{i j}^{\text {gap }}\right)^{2}$. However, equations (15)-(17) are used in the following study for the sake of simplicity.

When $R_{i}^{\text {chem }}$ and $R_{i}^{\text {gap }}$ are independent stochastic variables each other, $R_{i}=R_{i}^{\text {chem }}+g R_{i}^{\text {gap }}$ is also a stochastic variable. Therefore we obtain the following equations instead of equations (6)-(8).

$$
\begin{gathered}
x_{i}(n+1)=\theta\left(\sum_{j \in \mathcal{G}} w_{i j}^{\text {chem }} n_{i j}^{\text {chem }} x_{j}(n)+g \sum_{j \in \mathcal{G}} n_{i j}^{\text {gap }}\left(x_{j}(n)-x_{i}(n)\right)-C_{i}+R_{i}\right), \\
R_{i} \sim \mathrm{N}\left(\mu_{i}, \sigma_{i}^{2}\right), \\
\mu_{i}=\mu_{i}^{\text {chem }}+g \mu_{i}^{\text {gap }}, \\
\sigma_{i}^{2}=\left(\sigma_{i}^{\text {chem }}\right)^{2}+\left(g \sigma_{i}^{\text {gap }}\right)^{2} .
\end{gathered}
$$

When there is no prior knowledge on the probabilities $p_{i j}, q_{j}$ and $r_{i}$, we assume that $p_{i j}=1 / 2$, $q_{j}=1 / 2$ and $r_{i}=1 / 2$ for any $i$ and any $j$. That is, any $w_{i j}^{\text {chem }}\left(i \in \mathcal{G}, j \in \mathcal{G}^{\mathrm{c}}\right)$ takes +1 or -1 
with equal probability, any $x_{j}(n)\left(j \in \mathcal{G}^{\mathrm{c}}\right)$ takes 1 or 0 with equal probability, and any $x_{i}(n)$ in equation (8) takes 1 or 0 with equal probability. In this case, equations (12), (13), (16) and (17) become

$$
\begin{aligned}
\mu_{i}^{\text {chem }} & =0, \\
\left(\sigma_{i}^{\text {chem }}\right)^{2} & =\frac{1}{2} \sum_{j \in \mathcal{G}^{\mathrm{c}}}\left(n_{i j}^{\text {chem }}\right)^{2}, \\
\mu_{i}^{\text {gap }} & =0, \\
\left(\sigma_{i}^{\text {gap }}\right)^{2} & =\frac{1}{2} \sum_{j \in \mathcal{G}^{\mathrm{c}}}\left(n_{i j}^{\text {gap }}\right)^{2} .
\end{aligned}
$$

It must be emphasized that equation (18) is the closed equation of $x_{i}(n)(i \in \mathcal{G})$. We regard that the threshold $C_{i}$ is replaced with the effective threshold $\left(C_{i}-R_{i}\right)$ in equation (18).

\section{Application: Study of Synaptic Signs}

In this section, the presented formulation is applied to determine the synaptic signs of $C$. elegans. The touch-induced movement is adopted as behavioral criteria to determine the synaptic signs. That is, $w_{i j}^{\text {chem }}$ in equation (18) is determined to satisfy the touch-induced movement of C. elegans.

\subsection{Touch sensitivity circuit and behavioral criteria.}

The touch sensitivity circuit of $C$. elegans consists of three classes of sensory neurons, five classes of interneurons and five classes of motor neurons (Chalfie et al., 1985). The sensory neurons for anterior touch are ALM (ALMR/L) and AVM, while those for posterior touch are PLM (PLMR/L). The interneurons are PVC (PVCR/L), AVA (AVAR/L), AVB (AVBR/L), AVD (AVDR/L) and LUA (LUAR/L). The motor neurons for forward movement are VB (VB1VB11) and DB (DB1-DB7), while those for backward movement are VA (VA1-VA12), DA (DA1-DA9) and AS (AS1-AS11). Although LUA are the sensory neurons for posterior touch in Chalfie et al. (1985), we regard LUA as the interneurons following the description in White et al. (1986) and Wood (1988). When LUA are assumed to be the sensory neurons for posterior touch, we have confirmed that approximately the same results as those for LUA interneurons are obtained.

We set $\mathcal{G}$ and $\mathcal{G}^{\mathrm{c}}$ noted in Section 2 as follows.

$\mathcal{G}$ : The neurons which are mainly associated with the touch-induced movement. That is, ALM, AVM, PLM, PVC, AVA, AVB, AVD, LUA, VB, DB, VA, DA and AS neurons.

$\mathcal{G}^{\mathrm{c}}$ : The remaining neurons of $C$. elegans.

The database of the neural connectivity (Oshio et al., 1998) is used to determine $n_{i j}^{\text {chem }}$ and $n_{i j}^{\text {gap }}$. Oshio et al. (1998) have been derived from Albertson and Thomson (1976) and White et 
al. (1986) so that all the chemical synapses and all the gap junctions of C. elegans are exactly listed.

In the previous studies (Wicks et al., 1996; Majewska and Yuste, 2001), two reductions have been given in their neural circuits. One is reduction of the 'thin' chemical synaptic pathways. The other is reduction of the motor neurons. The former reduction is probably due to the database used in their studies. Wicks et al. (1996) and Majewska and Yuste (2001) have used the database (Achacoso and Yamamoto, 1992) in which all the synaptic connections of C. elegans are not listed exactly. The latter reduction is due to the evidence that many gap junctions exist between AVB neurons and the motor neurons for forward movement and between AVA neurons and the motor neurons for backward movement. Neurons coupled by a gap junction are often considered to be electrically conjugate since a gap junction propagates electrical signals in both directions instantaneously (Bennett and Spray, 1985). Therefore AVB interneurons have been responsible for forward movement and AVA interneurons have been responsible for backward movement in their studies. However, the gap junctions also exist between the sensory neuron and the interneuron and between the interneurons in the touch sensitivity circuit. In addition, many chemical synapses exist between the interneuron and the motor neuron. For these reasons, we consider the motor neurons in the touch sensitivity circuit and determine the synaptic signs between the interneuron and the motor neuron, which have not determined in the previous studies. All the neurons $\mathcal{G}$ and all the pathways between them are considered in the presented study.

The following behavioral criteria are adopted to determine the synaptic signs.

- Behavioral criterion (1): Normal touch response of C. elegans. That is, backward movement for anterior touch and forward movement for posterior touch.

- Behavioral criterion (2): Normal or lack of touch response for laser ablation of the neurons (Table III in Chalfie et al. (1985)). In the lesion table (Chalfie et al., 1985), the data of the uncoordinated movements and the habituation of the defective worms are not used as the behavioral criteria. It is not clearly decided how we should deal with the uncoordinated movements and the habituation in our simulation.

- Behavioral criterion (3): Forward movement for free locomotion without stimulation. When the worms move freely on the surface of agarose plates, the duration of forward movement is much longer than the durations of backward movement, resting and turns (Shingai, 2000).

The following procedures are carried out to study the synaptic signs. (i) The value of $w_{i j}^{\text {chem }}$ is set either as $w_{i j}^{\text {chem }}=+1$ (excitatory) or $w_{i j}^{\text {chem }}=-1$ (inhibitory). In the presented study, the Dale's principle is assumed for all neurons so that the synaptic signs are set to be equal irrespective of the postsynaptic neurons.

$$
w_{i j}^{\text {chem }}=w_{j}^{\text {chem }} \text { for any } i .
$$

Equation (26) indicates that the synaptic sign is assigned not to the chemical synaptic pathway but to the presynaptic neuron. (ii) The fixed random number is assigned to $R_{i}$. When "touch stimulus' is constantly added to the sensory neurons as the boundary condition, we assume that 
the influence of the neurons $\mathcal{G}^{\mathrm{c}}$ on the neurons $\mathcal{G}$ is also constant. This is the reason that $R_{i}$ is fixed in a time course of a simulation once a set of $R_{i}$ is initially given in the touch sensitivity circuit. Equation (19) is used to estimate the noise level. The Mersenne Twister algorithm (Matsumoto and Kurita, 1992, 1994; Matsumoto and Nishimura, 1998) is used to generate a pseudo-random number in a computer. (iii) A computer simulation is performed. When the behavioral criteria (1)-(3) are satisfied for a given configuration of $w_{i}^{\text {chem }}$ and $R_{i}$, we regard that the synaptic signs and the inputs from the neurons $\mathcal{G}^{\mathrm{c}}$ are plausible. These procedures are repeated for all the possible combinations of $w_{i}^{\text {chem }}$ with a number of samples for the sets of $R_{i}$.

We use the following conditions of our simulation. The initial condition is that $x_{i}(0)=0$ for any neuron. The motor neurons are reset in $x_{i}(n)=0$ at every time step since we assume that electrical signal is propagated from the motor neurons to the muscle instantaneously. As the boundary condition for anterior touch, ALM and AVM sensory neurons are fixed in the active state. As the boundary condition for posterior touch, on the other hand, PLM sensory neurons are fixed in the active state. As the boundary condition for the free locomotion, all the sensory neurons are fixed in the inactive state. For ablation of the selected neuron, all pathways from the ablated neuron are removed in the circuit. To decide whether 'forward movement' or 'backward movement' is caused or not in our simulation, the time course of the state of the motor neurons is observed. In the presented study, we decide that 'forward movement' is caused if VB and DB motor neurons are active for more than 7 time steps out of 10 time steps and VA, DA and AS motor neurons are inactive for more than 7 time steps out of 10 time steps. On the other hand, we decide that 'backward movement' is caused if VA, DA and AS motor neurons are active for more than 7 time steps out of 10 time steps and VB and DB motor neurons are inactive for more than 7 time steps out of 10 time steps.

We set the ratio $g$ and the threshold $C_{i}$ as follows. A gap junction provides a highconductance pathway for electrical current (Kandel et al., 2000). From the electrotonic characteristics of $C$. elegans, a conductance of the gap junction has been estimated as $g^{\text {gap }}=5 \mathrm{nS}$ (Wicks et al., 1996). On the other hand, a conductance of the chemical synapse is a graded function of presynaptic membrane potential $g^{\text {chem }}\left(V_{\text {pre }}\right)=g_{0}^{\text {chem }} f\left(V_{\text {pre }}\right)$ (Lockery and Sejnowski, 1992; DeSchutter et al., 1993). Here $g_{0}^{\text {chem }}$ is the maximum conductance, $V_{\text {pre }}$ is the presynaptic membrane potential and $f\left(V_{\text {pre }}\right)$ is a physiologically determined sigmoidal function of $V_{\text {pre }}$ $\left(0<f\left(V_{\text {pre }}\right)<1\right)$. Wicks et al. $(1996)$ have estimated $g_{0}^{\text {chem }}$ of $C$. elegans as $g_{0}^{\text {chem }}=0.6 \mathrm{nS}$. In the McCulloch-Pitts model, the graded function $g^{\text {chem }}\left(V_{\text {pre }}\right)$ is replaced by a step function such as $g^{\text {chem }}\left(V_{\text {pre }}\right)=g_{0}^{\text {chem }}$ if $V_{\text {pre }}>C_{i}$ and $g^{\text {chem }}\left(V_{\text {pre }}\right)=0$ if $V_{\text {pre }}<C_{i}$ (Cowan, 1990). Therefore $g=g^{\text {gap }} / g_{0}^{\text {chem }}=5 / 0.6$ is a reasonable value for $C$. elegans. In addition to $g=5 / 0.6$, we also study the cases of $g=1,2.5 / 0.6$ (half) and 10/0.6 (twice) to confirm the robustness of our results against a choice of $g$ in a wide range of parameter space. On the threshold $C_{i}$, we set $C_{i}=0$ for any neuron for the sake of simplicity. Although activation of the neuron strongly depends on the threshold in the McCulloch-Pitts model, too careful choice of $C_{i}$ might not be required in equation (18) since the Gaussian random number $R_{i}$ is added to $C_{i}$.

\subsection{Numerical results.}

Since the synaptic signs of the motor neurons are not determined owing to the boundary condition for the motor neurons, the synaptic signs $w_{i}^{\text {chem }}$ are determined for the remaining 
seven classes of neurons in the touch sensitivity circuit. Therefore there are $2^{7}$ possible configurations of $w_{i}^{\text {chem }}$. The number of random sampling of $R_{i}$ is $10^{8}$ for any configuration of $w_{i}^{\text {chem }}$. Consequently, $2^{7} \times 10^{8}$ configurations of $w_{i}^{\text {chem }}$ and $R_{i}$ are tested whether they satisfy the behavior criteria (1)-(3) or not. We single out functional configurations (functional circuits) which satisfy all behavior criteria out of $2^{7} \times 10^{8}$ samplings.

The number of the functional configurations of $w_{i}^{\text {chem }}$ and $R_{i}$ is shown in Table 1 . Since there are many functional configurations of $w_{i}^{\text {chem }}$ and $R_{i}$, the average synaptic signs $\left\langle w_{i}^{\text {chem }}\right\rangle$ and the average effective thresholds $\left(C_{i}-\left\langle R_{i}\right\rangle\right)$ are shown in Table 2. Among the seven classes of neuron, the synaptic signs are unambiguously determined for four classes of neurons. From Table 2, we find that PVC and AVD neurons are excitatory, and that (ALM, AVM) neurons are inhibitory. The AVB neurons might be inhibitory. Dent et al. (1997) and Lee et al. (1999) have proposed that the chemical synaptic pathway from AVM to AVB neurons is functionally inhibitory. Our synaptic sign of AVM neuron agrees with their suggestions. For PLM, AVA and LUA neurons, the synaptic signs are not uniquely determined.

The touch sensitivity circuit is shown in Fig. 1 on the basis of the results in Table 2. In Fig. 1 , a signal pathway for anterior touch is (ALM, AVM) $\rightarrow$ AVD $\rightarrow$ (VA, DA, AS). On the other hand, a signal pathway for posterior touch is $\mathrm{PLM} \rightarrow \mathrm{PVC} \rightarrow(\mathrm{VB}, \mathrm{DB})$. Interesting results in Fig. 1 are that the average effective thresholds of PVC and AVD neurons are negative. In the presented study, $\left(C_{i}-R_{i}\right)<0$ (effectively negative threshold) implies $R_{i}>0$ (excitatory inputs from the neurons $\mathcal{G}^{\mathrm{c}}$ ) because of $C_{i}=0$. These results suggest that both PVC and AVD neurons are kept active owing to their effectively negative thresholds. Therefore it seems that forward movement and backward movement are caused at the same time since PVC and AVD neurons are responsible for forward movement and backward movement, respectively. In spite of these contradictory situations, both the free locomotion and the touch-induced movement are well explained in the following ways. (i) When PVC and AVD neurons are active, AVB neurons become active owing to the excitatory inputs from both PVC and AVD neurons so that AVB neurons send the inhibitory signal to (DA, DA, AS) neurons. Consequently, (DA, $\mathrm{DA}, \mathrm{AS}$ ) neurons are inactive since the sum of the inhibitory input from AVB neurons and the excitatory inputs from both PVC and AVD neurons is less than the positive threshold of (DA, DA, AS) neurons (Table 2). Thus only forward movement is caused for the free locomotion. (ii) For anterior touch, PVC and AVB neurons are kept inactive owing to the inhibitory input from (ALM, AVM) neurons. In consequence, only the signal pathway for anterior touch works to cause backward movement. (iii) For posterior touch, forward movement is explained in the same way for the free locomotion.

To confirm the effect of the neurons $\mathcal{G}^{\mathrm{c}}$ in equation (18), we also study the case of $R_{i}=0$ (i.e., the inputs from the neurons $\mathcal{G}^{\mathrm{c}}$ to the touch sensitivity circuit are completely neglected). In other words, the random field $R_{i}$ is fixed at its average $\mu_{i}=0$ in equation (19). In the case of $R_{i}=0$, the behavioral criterion (3) is never satisfied since all the neurons are kept inactive under the boundary condition for the free locomotion. Therefore only the behavioral criteria (1) and (2) are adopted to determine the synaptic signs. However, no functional configuration of the synaptic signs is obtained in our simulation. Even when the Dale's principle is abandoned, the synaptic signs are not determined at the reasonable value $g=5 / 0.6$. To obtain a functional configuration of $w_{i j}^{\text {chem }}$, which satisfies the behavioral criteria but does not satisfy the Dale's 
principle, we find that $g$ must be smaller than half of the reasonable value $g=5 / 0.6$. Thus the neurons $\mathcal{G}^{\mathrm{c}}$ is considered to play some role in the touch-induced movement of $C$. elegans, and the careful treatment of the neurons $\mathcal{G}^{\mathrm{c}}$ is necessary for understanding the function of the partial neural circuit. In the touch sensitivity circuit (Fig. 1), gap junctions connect AVA interneurons with both the motor neurons for forward movement and those for backward movement. Because of these connectivities, 'forward movement' and 'backward movement' tend to be caused at the same time when AVA interneurons are active. To satisfy the behavioral criteria, therefore, AVA interneurons should have a large positive threshold $\left(C_{i}-R_{i}\right)$ to avoid activation (see the results shown in Table 2). Since $C_{i}=0$ in the presented study, no functional configurations are found without the external noise $\left(R_{i}=0\right)$. In the previous studies (Wicks et al., 1996; Majewska and Yuste, 2001), this problem has not been occurred since AVA interneurons are supposed to be the responsible neurons only for backward movement (Section 3.1 for details).

\subsection{Touch sensitivity circuit with additional neurons.}

How robust are our results in Section 3.2 against a choice of the neurons $\mathcal{G}$ ? To study this problem, we examine the synaptic signs when a class of neurons is added to the touch sensitivity circuit. We choose FLP, AVE, PHB, SAB,AVJ or SDQ as an additional class of neurons since they have many chemical synapses and gap junctions between the neurons in the touch sensitivity circuit. For each case, the standard deviations $\sigma_{i}^{\text {chem }}$ in equation (23) and $\sigma_{i}^{\text {gap }}$ in equation (25) are recalculated according to the modified neural circuitry. Average of the functional synaptic signs is shown in Table 3. The synaptic signs in Table 3 are practically the same as those in Table 2. We find that the functional synaptic signs are robust against a modification of the neural circuitry.

In quantitative comparison of Table 3 with Table 2, the average synaptic signs of AVB and AVD neurons are changed when AVE neurons are added to the touch sensitivity circuit. The results implicitly suggests that AVE neurons have some influence on the touch-induced movement of $C$. elegans. This influence of AVE neurons has been also suggested by the uncoordinated locomotion of the unc-4 mutant (White et al., 1992; Miller et al., 1992). For VA neurons of the unc-4 mutant, by contrast with the wild-type, both the chemical synapses from AVA, AVD and AVE neurons and the gap junction from AVA neurons are replaced with the gap junction from AVB neurons. In addition, the chemical synapses from PVC to VA neurons exist in the unc-4 mutant. As a result of the miswired neural connectivity, the unc-4 mutants curl up their bodies for anterior touch while they can normally move forward for posterior touch. In the numerical study on probability current in the whole neural circuit of $C$. elegans (Funabashi et al., 2001), AVE neurons have been also associated with the touch-induced movement.

\section{Conclusions}

In the paper, we present a mathematical framework for studying a large biological neural circuit. A partial, relatively small, circuit is mainly focused and the remaining part is taken into account as the 'external noise'. The average and the variance of the noise are appropriately 
evaluated by the neural connectivity. Thus the degree of freedom is effectively reduced in neural modeling.

As an example of the application of our method, the synaptic signs are determined for the chemical synapses in the touch sensitivity circuit of $C$. elegans. In addition to the synaptic sign in the model (18)-(25), the effective threshold of the neuron, which determines whether the signal transmits from the neuron to others or not, is also determined at the same time. This is an advantage of the presented formulation. From the results in Section 3.2, we find that the influence of the external noise (i.e., the neurons which do not belong to the touch sensitivity circuit) is important to determine the synaptic signs in the touch sensitivity circuit. Owing to the external noise incorporated into the neural modeling, the behavior of $C$. elegans is well explained by the partial neural circuit with the physiologically adequate values of the control parameter.

The functional signal pathways for the touch stimuli in Section 3.2 are basically consistent with those in Majewska and Yuste (2001). However, the synaptic signs of PVC $\rightarrow$ AVA, AVB $\rightarrow$ AVA and AVD $\rightarrow$ LUA pathways are the opposite. Since Majewska and Yuste (2001) have reduced the neural connectivity and do not assume the Dale's principle, the differences of the synaptic signs might be caused. Because of physiological reality, Wicks et al. (1996) have used the cable equation to describe synaptic transmission while we make use of the McCulloch-Pitts neuron for the sake of mathematical simplicity. Although the touch sensitivity circuit is slightly different from the tap withdrawal circuit (Wicks and Rankin, 1995), our results of the synaptic signs are consistent with those in Wicks et al. (1996). However, the signal pathways for the touch stimuli, which are suggested by the the functional synaptic signs in the tap withdrawal circuit, are different from our pathways. The differences might be caused by the reduction of the motor neurons (Section 3.1 for details). It is not clear that the cable equation without the external noise gives the same result as that in Wicks et al. (1996) when the motor neurons are considered in the tap withdrawal circuit.

The numerical result may depend on a choice of neural mode quantitatively. The McCullochPitts model is not the most suitable model to represent a real nervous system of $C$. elegans since the McCulloch-Pitts neuron is activated stepwisely. Prediction on the synaptic signs using a more physiologically relevant model is left for our future work.

\section{ACKNOWLEDGMENTS}

The authors would like to thank Yuko Osana for illuminating discussion which motivated the presented work. The authors are also grateful to Hiroshi Takano, Kiyoshi Kawamura, Kotaro Oka, Hiroaki Kagawa and Ryuzo Shingai for valuable comments and criticisms. The presented work was supported by the 'Research for the Future Program' from the Japan Society for the Promotion of Science (JSPS-RFTF 96I00102). 


\section{REFERENCES}

Achacoso, T. B. and W. S. Yamamoto (1992). AY's neuroanatomy of C. elegans for computation, Boca Raton, FL: CRC Press.

Alberts, B., A. Johnson, J. Lewis, M. Raff, K. Roberts and P. Walter (2002). Molecular Biology of the Cell, 4th edn., New York: Garland.

Albertson, D. G. and J. N. Thomson (1976). The pharynx of Caenorhabditis elegans. Phil. Trans. R. Soc. Lond. B 275, 299-325.

Bennett, M. V. L. and D. C. Spray (Eds) (1985). Gap Junctions, New York: Cold Spring Harbor Laboratory Press.

Cangelosi, A. and D. Parisi (1997). A neural network model of Caenorhabditis elegans: The circuit of touch sensitivity. Neural Processing Letters 6, 91-98.

Chalfie, M., J. E. Sulston, J. G. White, E. Southgate, J. N. Thomson and S. Brenner (1985). The neural circuit for touch sensitivity in Caenorhabditis elegans. J. Neurosci. 5, 956-964.

Cowan, J. D. (1990). McCulloch-Pitts and related neural nets from 1943 to 1989. Bull. Math. Biophys. 52, 73-97.

Dale, H. H. (1935). Pharmacology and nerve endings. Proc. Roy. Soc. Med. 28, 319-332.

DeSchutter, E., J. D. Angstadt and R. L. Calabrese (1993). A model of graded synaptic transmission for use in dynamic network simulations. J. Neurophysiol. 69, 1225-1235.

Dent, J. A., M. W. Davis and L. Avery (1997). avr-15 encodes a chloride channel subunit that mediates inhibitory glutamatergic neurotransmission and ivermectin sensitivity in Caenorhabditis elegans. EMBO. J. 16, 5867-5879.

Ferree, T. C. and S. R. Lockery (1999). Computational rules for chemotaxis in the nematode C. elegans. J. Comput. Neurosci. 6, 263-277.

Funabashi, Y., K. Kawamura, K. Oshio, S. Morita, Y. Osana, E. Akiyama and K. Oka (2001). Native response of C. elegans encoded in its neuron network. J. Phys. Soc. Jpn. 70, 11541161.

Kandel, E. R., J. H. Schwartz and T. M. Jessell (Eds) (2000). Principles of Neural Science, 4th edn, New York: McGraw-Hill.

Koch, C. and I. Segev (Eds) (1989). Methods in Neuronal Modeling, Cambridge, MA: MIT Press.

Langevin, P. (1908). Sur la theorie du mouvement Brownien. C. R. Acad. Sci. Paris 146, $530-533$.

Lee, R. Y., E. R. Sawin, M. Chalfie, H. R. Horvitz and L. Avery (1999). EAT-4, a homolog of a mammalian sodium-dependent inorganic phosphate cotransporter, is necessary for glutamatergic neurotransmission in Caenorhabditis elegans. J. Neurosci. 19, 159-167.

Lockery, S. R. and T. J. Sejnowski (1992). Distributed processing of sensory information in the leech. III. A dynamic neural network of the local bending reflex. J. Neurosci. 12, 3877-3895.

Majewska, A. and R. Yuste (2001). Topology of gap junction networks in C. elegans. J. Theor. Biol. 212, 155-167. 
Matsumoto, M. and Y. Kurita (1992). Twisted GFSR generators. ACM Trans. Modeling and Computer Simulation 2, 179-194.

Matsumoto, M. and Y. Kurita (1994). Twisted GFSR generators II. ACM Trans. Modeling and Computer Simulation 4, 254-266.

Matsumoto, M. \& Nishimura, T. (1998). Mersenne Twister: A 623-dimensionally equidistributed uniform pseudorandom number generator. ACM Trans. Modeling and Computer Simulation 8, 3-30.

McCulloch, W. S. and W. Pitts (1943). A logical calculus of the ideas immanent in nervous activity. Bull. Math. Biophys. 5, 115-133.

McIntire, S. L., E. Jorgensen, J. Kaplan and H. R. Horvitz (1993). The GABAergic nervous system of Caenorhabditis elegans. Nature 364, 337-341.

Miller, D. M., M. M. Shen, C. E. Shamu, T. R. Bürglin, G. Ruvkun, M. L. Dubois, M. Ghee and L. Wilson (1992). C. elegans unc-4 gene encodes a homeodomain protein that determines the pattern of synaptic input to specific motor neurons. Nature 355, 841-845.

Oshio, K., S. Morita, Y. Osana and K. Oka (1998). C. elegans synaptic connectivity data, Technical Report of CCEP, Keio Future, No.1, Keio University, Japan (Revised edition is appeared).

Riddle, D. L., T. Blumenthal, B. J. Meyer and J. R. Priess (Eds) (1997). C. elegans II, New York: Cold Spring Harbor Laboratory Press.

Shingai, R. (2000). Duration and frequencies of free locomotion in wild type and GABAergic mutants of Caenorhabditis elegans. Neurosci. Res. 38, 71-83.

Uhlenbeck, G. E. and L. S. Ornstein (1930). On the theory of Brownian motion. Phys. Rev. 36, 823-841.

White, J. G., E. Southgate, J. N. Thomson and S. Brenner (1986). The structure of the nervous system of the nematode Caenorhabditis elegans. Phil. Trans. R. Soc. Lond. B 314, 1-340.

White, J. G., E. Southgate and J. N. Thomson (1992). Mutations in the Caenorhabditis elegans unc-4 gene alter the synaptic input to ventral cord motor neurons. Nature 355, 838-841.

Wicks, S. R. and C. H. Rankin (1995). Integration of mechanosensory stimuli in Caenorhabditis elegans. J. Neurosci. 15, 2434-2444.

Wicks, S. R., C. J. Roehrig and C. H. Rankin (1996). A dynamic network simulation of the nematode tap withdrawal circuit: predictions concerning synaptic function using behavioral criteria. J. Neurosci. 16, 4017-4031.

Wood, W. B. (Ed.) (1988). The Nematoda Caenorhabditis elegans, New York: Cold Spring Harbor Laboratory Press. 
Table 1. The number of the functional configurations of $\left(w_{i}^{\text {chem }}, R_{i}\right)$ out of $2^{7} \times 10^{8}$ samplings.

\begin{tabular}{lccll}
\hline & \multicolumn{5}{c}{ the number of the functional configurations } \\
\cline { 2 - 5 } behavioral criteria & $g=1$ & $g=2.5 / 0.6$ & $g=5 / 0.6$ & $g=10 / 0.6$ \\
\hline$(1)$ & 1621757 & 105269 & 30886 & 6007 \\
$(1)$ and (2) & $268455(18)$ & $20557(13)$ & $14171(10)$ & $3777(20)$ \\
$(1),(2)$ and (3) & $196596(16)$ & $4665(11)$ & $1544(8)$ & $176(8)$ \\
\hline
\end{tabular}

The number of the functional configurations of $w_{i}^{\text {chem }}$ out of all $2^{7}$ possible combinations is shown in parentheses.

Table 2. Average synaptic sign and average effective threshold.

\begin{tabular}{lllll}
\hline & \multicolumn{3}{c}{ average synaptic sign and average effective threshold; $\left(\left\langle w_{i}^{\text {chem }}\right\rangle, C_{i}-\left\langle R_{i}\right\rangle\right)$} \\
\cline { 2 - 5 } neurons & \multicolumn{1}{c}{$g=1$} & \multicolumn{1}{c}{$g=2.5 / 0.6$} & \multicolumn{1}{c}{$g=5 / 0.6$} & \multicolumn{1}{c}{$g=10 / 0.6$} \\
\hline ALM, AVM & $(-1,-)$ & $(-1,-)$ & $(-1,-)$ & $(-1,-)$ \\
PLM & $(-0.06,-)$ & $(-0.76,-)$ & $(-0.78,-)$ & $(-0.85,-)$ \\
PVC & $(+1,-15.4 \pm 3.1)$ & $(+1,-6.9 \pm 5.6)$ & $(+1,-6.6 \pm 5.9)$ & $(+1,-6.1 \pm 5.4)$ \\
AVA & $(-0.04,57.0 \pm 39.8)$ & $(-0.24,97.5 \pm 36.0)$ & $(-0.21,155.9 \pm 42.7)$ & $(-0.14,259.6 \pm 43.8)$ \\
AVB & $(-1,-6.4 \pm 3.5)$ & $(-0.97,13.2 \pm 7.7)$ & $(-1,13.3 \pm 7.8)$ & $(-1,12.6 \pm 7.0)$ \\
AVD & $(+0.00,22.6 \pm 20.7)$ & $(+1,-4.8 \pm 5.2)$ & $(+1,-8.6 \pm 6.7)$ & $(+1,-20.1 \pm 12.7)$ \\
LUA & $(-0.12,0.5 \pm 6.4)$ & $(-0.37,6.5 \pm 11.2)$ & $(+0.20,6.6 \pm 20.7)$ & $(+0.41,9.2 \pm 35.4)$ \\
VB, DB & $(-, 5.3 \pm 3.9)$ & $(-, 7.3 \pm 4.4)$ & $(-, 9.6 \pm 6.4)$ & $(-, 15.0 \pm 9.6)$ \\
VA, DA, AS & $(-,-2.5 \pm 9.1)$ & $(-, 28.1 \pm 4.2)$ & $(-, 29.4 \pm 2.8)$ & $(-, 29.7 \pm 2.6)$ \\
\hline
\end{tabular}

The average synaptic sign $\left\langle w_{i}^{\text {chem }}\right\rangle>0$ or $\left\langle w_{i}^{\text {chem }}\right\rangle<0$ indicates that the synaptic sign is likely to be excitatory or inhibitory, respectively. Especially, $\left\langle w_{i}^{\text {chem }}\right\rangle=+1$ and $\left\langle w_{i}^{\text {chem }}\right\rangle=-1$ indicate that the synaptic sign is uniquely determined. The symbol '-' represents that the synaptic sign is not determined owing to the boundary conditions for the neurons. The standard deviation of $w_{i}^{\text {chem }}$ is not shown since $w_{i}^{\text {chem }}$ is either $w_{i}^{\text {chem }}=+1$ or $w_{i}^{\text {chem }}=-1$.

Table 3. Average synaptic sign in the touch sensitivity circuit with additional neurons; FLP, AVE, PHB, SAB,AVJ or SDQ. Parameter; $g=5 / 0.6$.

\begin{tabular}{lrrrrrr}
\hline & \multicolumn{6}{c}{ average synaptic sign; $\left\langle w_{i}^{\text {chem }}\right\rangle$} \\
\cline { 2 - 7 } neurons & FLP & AVE & PHB & SAB & AVJ & SDQ \\
\hline ALM, AVM & -1 & -1 & -1 & -1 & -1 & -1 \\
PLM & -0.36 & -0.28 & -0.65 & -0.39 & -0.38 & -0.75 \\
PVC & +1 & +1 & +1 & +1 & +1 & +1 \\
AVA & -0.00 & -0.01 & -0.14 & -0.01 & -0.01 & -0.25 \\
AVB & -1.00 & +0.40 & -1 & -0.48 & -1 & -1.00 \\
AVD & +1 & +0.41 & +1 & +1 & +1 & +1 \\
LUA & -0.31 & -0.15 & -0.02 & -0.77 & -0.46 & +0.19 \\
additional $X$ & +0.49 & -0.88 & +0.31 & 0 & -0.65 & -0.22 \\
\hline
\end{tabular}




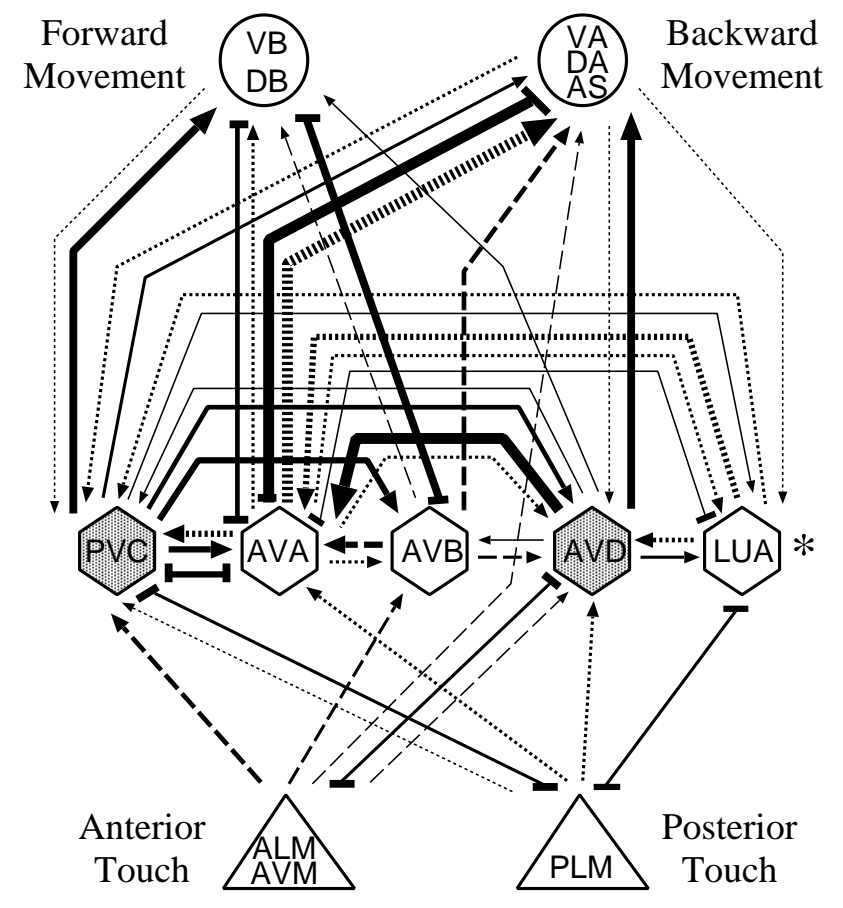

Figure 1. The touch sensitivity circuit of C. elegans. The circuit consists of three classes of sensory neurons (triangles), five classes of interneurons (hexagons) and five classes of motor neurons (circles). Gap junctions are represented by T lines $(-1)$. Excitatory synaptic connections are represented by solid arrows while inhibitory synaptic connections are represented by broken arrows. Dotted arrows represent that the synaptic sign is not uniquely determined. Shaded neurons have the effectively negative thresholds $\left(C_{i}-R_{i}<0\right)$ while unshaded neurons have the effectively positive thresholds $\left(C_{i}-R_{i}>0\right)$. The width of line is roughly proportional to the number of connections between the neurons. $g=5 / 0.6$. $\left(^{*}\right)$ The sign of the effective threshold is not identified for the LUA interneurons. 